\title{
COUNTABLY ADDITIVE HOMOMORPHISMS BETWEEN VON NEUMANN ALGEBRAS
}

\author{
L. J. BUNCE AND J. HAMHALTER
}

(Communicated by Palle E. T. Jorgensen)

\begin{abstract}
Let $M$ and $N$ be von Neumann algebras where $M$ has no abelian direct summand. A *-homomorphism $\pi: M \rightarrow N$ is said to be countably additive if $\pi\left(\sum_{1}^{\infty} e_{n}\right)=\sum_{1}^{\infty} \pi\left(e_{n}\right)$, for every sequence $\left(e_{n}\right)$ of orthogonal projections in $M$. We prove that a $*$-homomorphism $\pi: M \rightarrow N$ is countably additive if and only if $\pi(e \vee f)=\pi(e) \vee \pi(f)$ for every pair of projections $e$ and $f$ of $M$. A corollary is that if, in addition, $M$ has no Type $\mathrm{I}_{2}$ direct summands, then every lattice morphism from the projections of $M$ into the projections of $N$ is a $\sigma$-lattice morphism.
\end{abstract}

Let $M$ and $N$ be von Neumann algebras. A $*$-homomorphism $\pi: M \rightarrow N$ is said to be countably additive if $\pi\left(\sum e_{n}\right)=\sum \pi\left(e_{n}\right)$ for every sequence $\left(e_{n}\right)$ of orthogonal projections in $M$. Thus countable additivity is equivalent to weak-* continuity on all $\sigma$-finite von Neumann subalgebras of $M$.

It is well known $[3,8]$ (see also $[9, \mathrm{~V} .5 .1]$ ) that a $*$-homomorphism $\pi: M \rightarrow$ $N$ is automatically weak-* continuous when

(a) $M$ is Type $\mathrm{II}_{1}$ and $\sigma$-finite and $N$ acts on a separable Hilbert space, or

(b) $M$ is properly infinite and $M$ and $N$ are $\sigma$-finite.

It is worth noting that, in either case, it is not enough to know only that $M$ is $\sigma$-finite. For instance (see the argument of [9, V. 5.2], for example) when $M$ is Type II, with an infinite-dimensional centre, then $M$ has weak- * discontinuous factor representations; and when $M=B(H)$, where $H$ is a separable (or otherwise) Hilbert space, then each nontrivial representation of the Calkin algebra $B(H) / K(H)$ induces a weak-* discontinuous representation of $M$.

The purpose of this paper is to show that, free of all cardinality qualifications and all (except trivial) restrictions upon the type of the von Neumann algebras in question, a natural algebraic condition characterises countably additive *homomorphisms. We prove:

Theorem. Let $\pi: M \rightarrow N$ be a $*$-homomorphism between von Neumann algebras, where $M$ has no abelian direct summand. Then the following are equivalent.

Received by the editors May 2, 1994.

1991 Mathematics Subject Classification. Primary 46L50.

The second author would like to thank the Grant Agency of the Czech Republic for the support of his research (Grant No. 201/93/0953). 
(i) $\pi(e \vee f)=\pi(e) \vee \pi(f)$, for all projections $e$ and $f$.

(ii) $\pi$ is countably additive.

We remark that the Theorem fails when $M$ is abelian and infinite dimensional because then, as is easy to see, condition (ii) need not be true whereas (i) is automatically satisfied.

The naturality of the condition (i) of the Theorem may be seen through the axiomatic foundations of quantum mechanics. Given projections $e$ and $f$ of $M$ we have $[e+1-(e \vee f)] \vee[f+1-(e \vee f)]=1$. Thus in the case when $\pi(1)=1$, the condition (i) is equivalent to (for projections $e$ and $f$ of $M$ )

$$
e \vee f=1 \text { implies } \pi(e) \vee \pi(f)=1 \text {. }
$$

This has the following completely natural physical interpretation:

the truth of the "proposition" " $e$ or $f$ " implies the truth of " $\pi(e)$ or $\pi(f)$ ".

Thus, apart from any independent mathematical interest, the Theorem might find some use in the foundations of quantum mechanics.

We need two preparatory lemmas.

Lemma 1. Let $\rho_{1}$ be a singular state of $l^{\infty}$, and let $\rho_{2}$ be a pure state of $M_{n}(\mathbb{C})$, where $2 \leq n<\infty$. Then there exist projections $e$ and $f$ in $l^{\infty} \otimes M_{n}(\mathbb{C})$ for which $e \vee f=1$ and $\left(\rho_{1} \otimes \rho_{2}\right)(e)=\left(\rho_{1} \otimes \rho_{2}\right)(f)=0$.

Proof. This was proved in [5, Example 3.2]. See also [1, Lemma 1].

Lemma 2 (Fillmore [4], Kadison [6]). Let $M$ be a von Neumann algebra, and let $\left(f_{n}\right)$ be a sequence of orthogonal projections in $M$.

(i) If $M$ is Type $\mathrm{I}_{n}$, where $2 \leq n<\infty$, then there exist orthogonal and equivalent projections $e_{1}, \ldots, e_{n}$ in $M$ with sum 1 such that $e_{i} f_{j}=$ $f_{j} e_{i}$, for all $i$ and $j$.

(ii) If $M$ has no finite Type I direct summand, then there exists in $M a$ projection $e \sim 1-e$ such that $e f_{j}=f_{j} e$, for all $j$.

Proof. The cases of (i) and, when $M$ is Type $\mathrm{II}_{1}$, (ii) are proved in [6, Lemma 3.6 and Corollary 3.15]. As the von Neumann subalgebra of $M, W^{*}\left(\left\{f_{n}: n \in\right.\right.$ $\mathbb{N}\}) \simeq l^{\infty}$, generated by the $f_{n}$ is singly generated, the case of (ii) when $M$ is properly infinite follows immediately from [4, Theorem 3].

Proof of Theorem. (ii) $\Rightarrow$ (i). Let the *-homomorphism $\pi: M \rightarrow N$ be countably additive, and let $e$ and $f$ be projections in $M$. Then $\pi(e) \vee \pi(f) \leq$ $\pi(e \vee f)$. Let $\rho$ be a normal state of $N$ such that $\rho(\pi(e) \vee \pi(f))=0$. By spectral theory, there is an increasing sequence $\left(p_{n}\right)$ of projections in $M$ converging strongly to $e \vee f$ where, for each $n, e+f \geq \alpha_{n} p_{n}$ for some real number $\alpha_{n}>0$. But then

$$
\rho \pi(e \vee f)=\rho \lim \pi\left(p_{n}\right)=\lim \rho \pi\left(p_{n}\right)=0 .
$$

Therefore $\pi(e) \vee \pi(f)=\pi(e \vee f)$.

(i) $\Rightarrow$ (ii). Assume (i) and, without loss of generality, that $\pi(1)=1$. Suppose first that $M$ contains orthogonal and equivalent projections $e_{1}, \ldots, e_{n}$ such that $e_{1}+\cdots+e_{n}=1$, for some $n$ where $2 \leq n<\infty$. Put $e=e_{1}$. Via a suitable choice of $n \times n$ matrix units $\left(u_{i j}\right)$, there is (see [9, IV 1.8]) a surjective *-isomorphism

$$
\varphi: M \rightarrow(e M e) \otimes M_{n}(\mathbb{C})
$$


In turn $\left(\pi\left(u_{i j}\right)\right)$ is a system of $n \times n$ matrix units for $N$, inducing surjective *-isomorphism

$$
\psi: N \rightarrow(f N f) \otimes M_{n}(\mathbb{C})
$$

where $f=\pi(e)$, and resulting in the commutative diagram

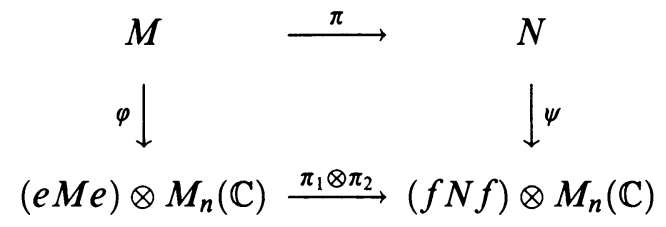

where $\pi_{1}: e M e \rightarrow f N f$ satisfies $\pi_{1}(a) \otimes 1_{n}=\psi \pi(a)$ and $\pi_{2}$ is an automorphism of $M_{n}(\mathbb{C})$. In particular (as $\psi$ is normal, being an isomorphism), we see that $\pi \mid e M e$ is countably additive if $\pi_{1}$ is countably additive. We prove the latter.

In order to derive a contradiction, suppose that $\pi_{1}$ is not countably additive. Then there is a sequence $\left(p_{n}\right)$ of orthogonal projections in $e M e$ such that

$$
\sum_{1}^{\infty} p_{n}=e \text { and } \sum_{1}^{\infty} \pi_{1}\left(p_{n}\right) \neq \pi_{1}(e)=f .
$$

Choose a normal state $\rho_{1}$ of $f N f$ such that $\rho_{1}\left(\sum_{1}^{\infty} \pi_{1}\left(p_{n}\right)\right)=0$ and let $\rho_{2}$ be a pure state of $M_{n}(\mathbb{C})$. Then $\rho_{1} \otimes \rho_{2}$ is a normal state of $(f N f) \otimes M_{n}(\mathbb{C})$. In addition, $\rho_{1} \pi_{1}$ is singular on the von Neumann subalgebra of $M, W^{*}\left(\left\{p_{n}: n \in\right.\right.$ $\mathbb{N}\}) \simeq l^{\infty}$, generated by the $p_{n}$ and $\rho_{2} \pi_{2}$ is a pure state of $M_{n}(\mathbb{C})$. Hence, by Lemma 1 applied to the state

$$
\left(\rho_{1} \otimes \rho_{2}\right)\left(\pi_{1} \otimes \pi_{2}\right)=\left(\rho_{1} \pi_{1}\right) \otimes\left(\rho_{2} \pi_{2}\right),
$$

there exist projections $p$ and $q$ in $(e M e) \otimes M_{n}(\mathbb{C})$ such that

$$
p \vee q=1 \quad \text { and }\left(\rho_{1} \otimes \rho_{2}\right)\left(\pi_{1} \otimes \pi_{2}\right)(p)=\left(\rho_{1} \otimes \rho_{2}\right)\left(\pi_{1} \otimes \pi_{2}\right)(q)=0 .
$$

Putting now $g=\varphi^{-1}(p)$ and $h=\varphi^{-1}(q)$ we have that $g \vee h=1$ so that $\pi(g) \vee \pi(h)=1$, by assumption. On the other hand $\tau=\left(\rho_{1} \otimes \rho_{2}\right) \psi$ is a normal state of $N$ with $\tau \pi(g)=\tau \pi(h)=0$, so that $\tau(\pi(g) \vee \pi(h))=0$ in contradiction.

This proves that $\pi$ is countably additive on $e_{i} M e_{i}$, for each $i=1, \ldots, n$.

We will proceed to establish (ii) on a case-by-case basis. Let $\left(f_{n}\right)$ be a sequence of orthogonal projections in $M$.

(a) If $M$ is Type $I_{n}$, where $2 \leq n<\infty$ then, by part (i) of Lemma 2, there exist orthogonal and equivalent projections $e_{i}$ in $M$ with $e_{1}+\cdots+e_{n}=1$ such that $e_{i} f_{j}=f_{j} e_{i}$ for all $i$ and $j$. Since, for each $i=1, \ldots, n, \pi$ is countably additive (by the above) on $e_{i} M e_{i}$ and $\left(e_{i} f_{n}\right)$ is an orthogonal sequence of projections in $e_{i} M e_{i}$, we have

$$
\pi\left(e_{i}\right) \pi\left(\sum_{1}^{\infty} f_{n}\right)=\pi\left(e_{i} \sum_{1}^{\infty} f_{n}\right)=\pi\left(e_{i}\right) \sum_{1}^{\infty} \pi\left(f_{n}\right),
$$

from which it follows that $\pi$ is countably additive on $M$.

(b) If $M$ is finite Type $\mathrm{I}$, then $M$ is an $l^{\infty}$ direct sum of countably many algebras of the form Type $I_{n}$, where $2 \leq n<\infty$. So, in view of (a), it is clearly enough in this case to show that $\pi$ is countably additive on the centre, 
$Z(M)$, of $M$. We can always choose projections $e_{1}, e_{2}, e_{3}$ and $f$ in $M$ so that $e_{1}+e_{2}+e_{3}=1$ with

$$
e_{1} \sim e_{2} \text { and } e_{3} \sim f \leq e_{1}+e_{2} .
$$

Now the first part of the proof applied to each of these equivalences, in turn, implies that $\pi$ is countably additive on $e_{i} M e_{i}$ and hence on $Z(M) e_{i}$, for $e=1,2,3$. It follows that $\pi$ is countably additive on $Z(M)$, as required.

(c) Finally, when $M$ is without finite Type I direct summands, part (ii) of Lemma 2 ensures the existence in $M$ of a projection $e \sim 1-e$ commuting with all of the $f_{n}$ and a calculation similar to that performed in (a) establishes countable additivity.

This completes the proof.

If $M$ is von Neumann algebra, then let $P(M)$ denote the projection lattice of $M$. Let $M$ and $N$ be von Neumann algebras. A function $\varphi: P(M) \rightarrow P(N)$ is said to be a lattice morphism if

(i) $\varphi(e+f)=\varphi(e)+\varphi(f)$, whenever $e$ and $f$ are orthogonal;

(ii) $\varphi(e \vee f)=\varphi(e) \vee \varphi(f)$, for all $e$ and $f$.

A lattice morphism $\varphi: P(M) \rightarrow P(N)$ is called a $\sigma$-morphism if

$$
\varphi\left(\bigvee_{1}^{\infty} e_{n}\right)=\bigvee_{1}^{\infty} \varphi\left(e_{n}\right) \text { for every sequence }\left(e_{n}\right)
$$

One consequence (Corollary 2) of the Theorem is that a lattice morphism $\varphi: P(M) \rightarrow P(N)$ is nearly always a $\sigma$-morphism.

A *-Jordan homomorphism $\pi: M \rightarrow N$ is a *-linear map where $\pi\left(x^{2}\right)=$ $\pi(x)^{2}$, for all $x$ in $M$. By a result of Størmer [7, Theorem 3.3] each *Jordan homomorphism between von Neumann algebras is the sum of a *homomorphism and a $*$-antihomomorphism. So, the Theorem, being valid for *-homomorphism and hence (trivially) for *-antihomomorphisms, is therefore valid for *-Jordan homomorphisms too. In other words, we have:

Corollary 1. If $\pi: M \rightarrow N$ is a *-Jordan homomorphism between von Neumann algebras $M$ and $N$ where $M$ has no abelian direct summands, then the following are equivalent.

(i) $\pi(e \vee f)=\pi(e) \vee \pi(f)$ for all projections $e$ and $f$.

(ii) $\pi$ is countably additive.

Corollary 2. Let $M$ and $N$ be von Neumann algebras where $M$ has neither abelian nor Type $\mathrm{I}_{2}$ direct summands. Then every lattice morphism $\varphi: P(M) \rightarrow$ $P(N)$ is a $\sigma$-morphism.

Proof. A lattice morphism $\varphi: P(M) \rightarrow P(N)$ extends to a *-Jordan homomorphism $\pi: M \rightarrow N$ (see [2, Proposition and Corollary 1], for example) which, by Corollary 1 , is countably additive. So $\varphi$ must be a $\sigma$-morphism.

\section{REFERENCES}

1. A. Amann, Jauch-Piron states in $W^{*}$-algebra quantum mechanics, J. Math. Phys. 28 (1987), 2384-2389.

2. L. J. Bunce and J. D. M. Wright, On Dye's Theorem for Jordan operator algebras, Exposition. Math. 11 (1993), 91-95. 
3. J. Feldman and J. M. G. Fell, Separable representations of rings of operators, Ann. of Math. (2) 65 (1957), 241-249.

4. P.A. Fillmore, On products of symmetries, Canad. J. Math. 18 (1966), 897-900.

5. J. Hamhalter, Pure Jauch-Piron states on von Neumann algebras, Ann. Inst. H. Poincaré Phys. Théor. 58 (1993), 173-187.

6. R. V. Kadison, Diagonalising matrices, Amer. J. Math. 106 (1984), 1451-1468.

7. E. Størmer, On the Jordan structure of $C^{*}$-algebras, Trans. Amer. Math. Soc. 120 (1965), 438-447.

8. M. Takesaki, On the non-separability of singular representation of operator algebra, Kodai Math. Sem. Rep. 12 (1960), 102-108.

9. _ـ Theory of operator algebras I, Springer, Berlin and New York, 1979.

Department of Mathematics, Reading University, P.O. Box 220, Reading RG6 2AX, ENGLAND

Department of Mathematics, Technical University of Prague, Technicka 2, 16627 Prague 6, Czech Republic 